01

\title{
Оптимальная пара ридберговских атомов щелочных металлов в несимметричных пеннинговских процессах ионизации
}

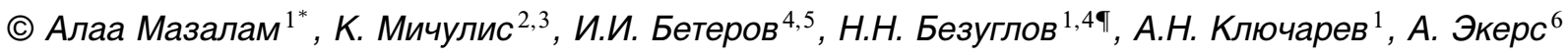 \\ ${ }^{1}$ Санкт-Петербургский государственный университет, \\ 199034 Санкт-Петербург, Россия \\ ${ }^{2}$ University of Latvia, Institute of Atomic Physics and Spectroscopy, \\ LV-1586 Riga, Latvia \\ ${ }^{3}$ Национальный исследовательский ядерный университет „МИФИ“, \\ 115409 Москва, Россия \\ ${ }^{4}$ Институт фризики полупроводников им. А.В. Ржанова Сибирского отделения РАН, \\ 630090 Новосибирск, Россия \\ ${ }^{5}$ Новосибирский государственный университет, \\ 630090 Новосибирск, Россия \\ ${ }^{6}$ King Abdullah University of Science and Technology (KAUST), \\ Computer, Electrical and Mathematical Science and Engineering Division (CEMSE), \\ Thuwal 23955-6900, Saudi Arabia \\ I e-mail: nikolay.bezuglov@gmail.com
}

Поступила в редакцию 05.02.2019 г.

В окончательной редакции 05.02.2019 г.

Принята к публикации 23.04.2019 г.

Исследованы особенности процессов пеннинговской ионизации (ПИ) в холодных газовых средах ридберговских атомов щелочных металлов. В отличие от атома водорода соответствующие автоионизационные ширины демонстрируют резкую зависимость (на порядки величин) от орбитальных квантовых чисел атомов, подверженных дальнодействующему диполь-дипольному взаимодействию. Важной чертой ПИ оказывается нетривиальная зависимость ее эффективности от размеров ридберговских частиц. Для всех видов атомов щелочных металлов нами найдены оптимальные, сильно асимметричные конфигурации ридберговских пар, которые приводят к взрывной интенсификации (на несколько порядков величин) образования свободных электронов за счет процессов ПИ. Это свойство делает ПИ важным источником образования первичных заряженных частиц при формировании холодной ридберговской плазмы. Приведенные численные данные для атомных пар калия демонстрируют существенное влияние резонанса Фёрстера (Förster) на значения констант скоростей ПИ.

Ключевые слова: ультрахолодные газовые среды, ридберговская атомная пара, пеннинговские автоионизационные ширины, холодная плазма.

DOI: $10.21883 /$ OS.2019.09.48186.45-19

\section{1. Введение}

Лазерные методы манипулирования квантовыми состояниями атомных систем являются важнейшей составляющей современных спектроскопических исследований с широкой областью применения для прикладных научных задач [1]. Прежде всего стоит отметить обширную тематику холодных ридберговских сред [2], которые в настоящее время представляются перспективными физическими объектами для решения проблем квантовой обработки информации [3]. Основной особенностью ридберговских систем являются их чрезвычайно большие размеры $\sim n^{2}$ (где $n$ обозначает главное квантовое число) и, как следствие, огромные дипольные моменты. Поэтому ридберговскими состояниями нейтральных атомов сравнительно легко управлять с помощью когерентного излучения [4], дополненного дальнодействующим

* Постоянное место работы: Physics Department, Faculty of Science, Menoufia University, Menoufia, Egypt. межатомным дипольным взаимодействием в сочетании с эффектами дипольной блокады [5]. Все это позволяет как конструировать $Q$-битные состояния на основе сверхтонкой структуры основных стабильных уровней холодных атомов щелочных металлов [6], так и выполнять при использовании внешних управляющих полей основные базовые квантовые операции [7].

Развитие вторичных радиационных или столкновительных процессов приводит, однако, к перемешиванию состояний, что может вызывать неуправляемое нарушение первичной селективности. Холодные ридберговские среды при определенных условиях быстро эволюционируют в холодную нейтральную плазму с формированием свободных электронов и протеканием разнообразных явлений типа спонтанного расширения плазмы, рекомбинации ридберговских атомов, плазменных неустойчивостей, распространения коллективных волн [8]. Например, в холодном ридберговском газе атомов рубидия, возбужденных в первоначальные со- 
стояния с главным квантовым числом $n_{0} \approx 50$ и концентрацией $\rho_{0}>5 \cdot 10^{10} \mathrm{~cm}^{-3}$, наблюдается спонтанный переход ридберговской среды в холодную плазму за время порядка $100 \mathrm{~ns}$ [9]. Этот переход осуществляется через так называемую „диффузионную“ стадию эволюции холодного газа с перераспределением энергии возбуждения ридберговских состояний и созданием асимметричных атомных пар, обладающих разными главными $n$ и орбитальными $l$ квантовыми числами.

Механизмы рождения свободных электронов в ультрахолодном газе еще не вполне ясны, однако физика ультранизкотемпературной плазмы наряду с традиционными столкновительными ионизационными или рекомбинационными процессами [10] должна учитывать образование заряженных частиц как за счет ионизации одиночных ридберговских атомов тепловым излучением [11], так и в результате пеннинговской автоионизации ридберговских пар [12]. Специфической чертой ультрахолодных сред является практическая неизменность межъядерных атомных расстояний $R$ на характерных экспериментальных временах измерений (несколько микросекунд). Поэтому скорости процессов, вызванных дальнодействующими потенциалами (типа диполь-дипольного и вандер-ваальсова), определяются соответствующими автоионизационными ширинами $\Gamma$ квазимолекулы $\{A+B\}$, составленной из взаимодействующих частиц $A$ и $B$. К наиболее важным механизмам межатомной ионизации относятся ассоциативный и пеннинговский [13]. Ассоциативную ионизацию (АИ) следует считать сравнительно малоэффективной [14] в холодных средах, поскольку, являясь пороговой реакцией [15], она может не обеспечиваться низкоэнергетическим тепловым движением атомов. Надпороговая пеннинговская ионизация (ПИ) ридберговских частиц обусловлена сильным дальнодействующим дипольным взаимодействием, в результате чего один из атомов $A\left(n_{i} l_{i}\right)$ ионизуется, а другой $A\left(n_{d} l_{d}\right)$ девозбуждается с сохранением суммарной энергии частиц, как это показано ниже на рис. 1.

В работе [16] рассматривались симметричные $\left(\left\{n_{i} l_{i}\right\}=\left\{n_{d} l_{d}\right\} \equiv\left\{n_{0} l_{0}\right\}\right) \quad$ процессы ПИ в широких диапазонах возбуждения $n_{0}=10-100$ и концентраций $\rho=10^{8}-10^{11} \mathrm{~cm}^{-3}$ ридберговских состояний атома рубидия. Как оказалось, соответствующие пеннинговские автоионизационные ширины $\Gamma_{n_{0} n_{0}}^{(\mathrm{sym})}$ не превышают значений $10 \mathrm{~s}^{-1}$. Это существенно ниже характерных значений констант скоростей $\sim W_{b l} \sim 10^{3} \mathrm{~s}^{-1}$ ионизации излучением черного тела [11]. Ситуация, однако, становится другой в случае несимметричных пар $\left(n_{i} \neq n_{d}\right)$. Согласно результатам работы [12], полученным для атомов водорода, при каждом фиксированном значении $n_{i}$ ионизирующегося атома существует оптимальное значение $\quad n_{d}^{\text {(opt) }}=2^{1 / 3} n_{i}^{2 / 3} \ll n_{i} \quad$ девозбуждающегося атома, для которого достигается максимальная величина $\Gamma_{n_{d} n_{i}}^{(\text {opt }}$ автоионизационной ширины атомарной пары $n_{i}, n_{d}$ :

$$
R^{6} \Gamma_{n_{d} n_{i}}^{(\mathrm{opt})}=\frac{9 \cdot 2^{11 / 3}}{17 \cdot \pi^{3}}\left(\frac{2^{2 / 3} \Gamma(2 / 3)}{3^{1 / 3}}\right)^{4} n_{i}^{19 / 3} .
$$

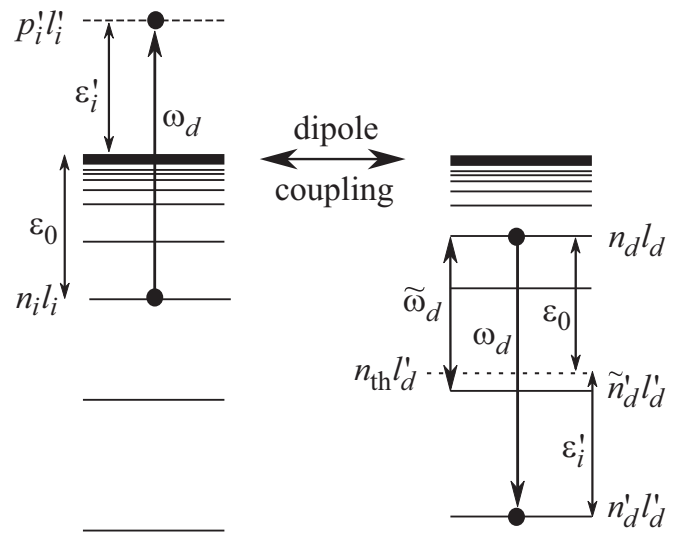

Рис. 1. Схематическое изображение оже-процесса для резонансного взаимодействия ридберговской атомной пары $\left\{A_{i}^{* *}, A_{d}^{* *}\right\}$ с континуумом. В случае несимметричных пар энергии связи ридберговских электронов в атомах различаются.

При этом $\Gamma_{n_{d} n_{i}}^{(\mathrm{opt})}$ в $n_{i}$ превышает значение $\Gamma_{n_{i} n_{i}}^{(\mathrm{sym})}$ для симметричной пары $\left\{n_{i} n_{i}\right\}$. В результате формирования несимметричных пар $\left(n_{d}<n_{0}<n_{i}\right)$ на ,диффузионной“ стадии эволюции ридберговского газа в холодную плазму [9] скорость ПИ взрывным образом возрастает на несколько порядков величин, и для определенных условий перекрывает эффекты ионизации за счет фонового излучения черного тела [12].

В случае атомов щелочных металлов пеннинговские ширины могут существенно (для лития на два порядка) отличаться от значений, предсказываемых эталонными „водородными“ формулами, полученными в работе [12]. Это связано с резкой зависимостью констант скоростей радиационных процессов от квантовых дефектов $\delta_{l}$, входящих в эффективные квантовые числа $n^{*}=n-\delta_{l}$ ридберговских состояний. В настоящей работе исследована (аналитически и численно) специфика протекания процессов ПИ в холодных газовых средах щелочных атомов. Пеннинговская ионизация для последних как качественно, так и количественно обладает большим многообразием особенностей по сравнению со случаем водородных атомов. Везде в дальнейшем используется атомная система единиц.

\section{2. Константы скоростей пеннинговской ионизации}

Количественное описание констант скоростей ПИ удобно проводить в рамках модифицированной модели Смирнова-Фирсова-Катцууры $[17,18]$. В данной модели основной динамической переменной является автоионизационная ширина $\Gamma_{N}=\tilde{\Gamma}_{N} / R^{6}$ пары сталкивающихся атомов. Если атомы находятся в квантовых состояниях $N \equiv\left\{N_{d}=n_{d} l_{d}, N_{i}=n_{i} l_{i}\right\}$ с фиксированным межъядерным расстоянием $R$, то приведенная пеннинговская ширина $\tilde{\Gamma}_{N}$ складывается из парциальных ширин $\tilde{\Gamma}_{N N}$, 
соответствующих индивидуальным связано-свободным переходам $N \rightarrow N^{\prime}$ в атомах (рис. 1):

$$
\begin{gathered}
\tilde{\Gamma}_{N} \equiv R^{6} \Gamma_{N}=\sum_{n_{d}^{\prime} l_{d}^{\prime} l_{i}^{\prime}} \tilde{\Gamma}_{N N^{\prime}}, \\
\tilde{\Gamma}_{N N^{\prime}}=\frac{c \sigma_{p h}\left(\varepsilon_{i}^{\prime}\right)}{\pi\left|\omega_{n_{d} l_{d}^{\prime} l_{d}^{\prime}}^{\prime}\right|} \frac{\left|D_{N_{D}^{\prime} N_{D}}\right|^{2}}{2 l_{d}+1} .
\end{gathered}
$$

В ширины $\tilde{\Gamma}_{N N^{\prime}}$ входят приведенные дипольные матричные элементы $D_{N_{D}^{\prime} N_{D}}$ для связанных переходов $d$-атома и сечения $\sigma_{p h}$ фотоионизации для $i$-атома в соответствии с тем, что его конечные состояния $N_{i}^{\prime}=p_{i}^{\prime} l_{i}^{\prime}$, характеризуемые импульсом $p_{i}^{\prime}$ образовавшегося свободного электрона, лежат в континууме энергий.

Сам механизм передачи электронной энергии из состояния $N$ в состояние $N^{\prime}$ ридберговской двухатомной системы (квазимолекулы) обусловливается резонансным оже-процессом (рис. 1). Частота $\omega_{d}=\omega_{n_{d} l_{d}}^{n_{d}^{\prime} l_{d}^{\prime}}$ парциальных атомных переходов определяет энергию, которую $d$-атом передает $i$-атому (рис. 1) за счет диполь-дипольного взаимодействия. Условие ионизации означает $\omega_{d} \geq \varepsilon_{0}$, где $\varepsilon_{0}=1 /\left(2 n_{i}^{* 2}\right)-$ энергия связи $i$-атома. Штрихованная линия на рис. 1 показывает положение воображаемого (виртуального) уровня, который лежит ниже состояния $n_{d} l_{d}$ на величину $\varepsilon_{0}$. Он, следовательно, определяет границу порога ионизации: суммирование в ряде (1) должно проводиться по значениям $n_{d}^{\prime} \leq n_{h}$, т.е., начиная с целого числа $\tilde{n}_{d}^{\prime}$, которое является ближайшим снизу к $n_{\text {th }}$.

Для применимости соотношений (2) необходимо выполнение следующих условий: (i) электронные оболочки атомов не перекрываются, т.е. межъядерное расстояние $R$ превосходит характерные размеры $n^{* 2}$ ридберговских состояний, (ii) атомы в холодной среде расположены хаотически и имеют однородное распределение возбужденных состояний по зеемановским подуровням $m$ (отсутствие ориентации и выстраивания).

Отметим, что для диполь-дипольного взаимодействия приведенная ширина $\tilde{\Gamma}_{N}(2)$ не зависит от межъядерного расстояния $R$. Она удобна для определения эффектов ПИ в ультрахолодном (,замороженном“") ридберговском газе при неизменных $R$. В обычных тепловых и субтепловых условиях необходимо учитывать относительную скорость $v$ сталкивающихся частиц, а константы скоростей реакций находить из соответствующих сечений. В модели Смирнова-Фирсова-Катцууры сечение ПИ, обусловленное диполь-дипольным взаимодействием, непосредственно связано с автоионизационной шириной $\tilde{\Gamma}_{N}[18]$ :

$$
\sigma_{\mathrm{PI}}=5.00 \cdot \tilde{\Gamma}_{N}^{2 / 5} / v^{2.5}
$$

\section{1. Квазиклассическое приближение}

Входящие в (2) характеристики $D_{N_{D}^{\prime} N_{D}}, \sigma_{p h}$ одноэлектронных оптических переходов определяются величина- ми интегралов перекрытия

$$
R_{n l}^{n^{\prime} l^{\prime}}=\int R_{n l} R_{n^{\prime} l^{\prime}} r^{3} d r ; \quad R_{n l}^{p^{\prime} l^{\prime}}=\int R_{n l} R_{p^{\prime} l^{\prime}} r^{3} d r
$$

для радиальных волновых функций связанных $\left(R_{n^{\prime} l^{\prime}}, R_{n l}\right)$ или свободных $\left(R_{p^{\prime} l^{\prime}}\right)$ электронных состояний из следующих представлений [19]:

$$
\begin{gathered}
D_{n^{\prime} l^{\prime}, n l}=\sqrt{\frac{l_{\max }}{2 l+1}} R_{n l}^{n^{\prime} l^{\prime}}, \quad l_{\max }=\max \left\{l, l^{\prime}\right\}, \\
\sigma_{p h}\left(n l \rightarrow p^{\prime} l^{\prime}\right)=\frac{4 \pi^{2} \omega_{n l}^{p^{\prime} l^{\prime}}}{3 c} \frac{l_{\max }}{2 l+1} \frac{1}{p^{\prime 3}}\left|R_{n l}^{p^{\prime} l^{\prime}}\right|^{2} .
\end{gathered}
$$

Расчеты радиальных интегралов выполняются в основном численными методами. Альтернативный подход связан с применением достаточно точных квазиклассических представлений [20-23]. В работе [23] для ридберговских состояний щелочных атомов получены сравнительно простые формулы для $R_{n l}^{n^{\prime} l^{\prime}}$. В настоящей работе мы воспользовались улучшенными (за счет дополнительных квантовых поправок) аналитическими представлениями $R_{n l}^{n^{\prime} l^{\prime}}$, резюме по которым приведено в Приложении.

Точность соответствующих квазиклассических формул зависит от разности $\gamma=n^{* *}-n^{*}$. При небольших $|\gamma|$ и $n^{*}>10$ соотношения (П1), (П2) приводят к ошибкам расчетов $R_{n l}^{n^{\prime} l^{\prime}}$ в доли процентов, в то время как асимптотическое продолжение $R_{n l}^{n^{\prime} l^{\prime}}$ в область больших $|\gamma|$ (формулы (П5),(П6)) обеспечивают ошибки порядка $2 \%$. Формулы (П5),(П6) позволяют также находить радиальные интегралы и для связанно-свободных переходов. Получаемая при этом точность проиллюстрирована на рис. 2 на примере расчета пороговых величин сечений фотоионизации $\sigma_{p h}(p=0)(5)$. Необходимые для вычислений $\sigma_{p h}$ значения квантовых дефектов ридберговских

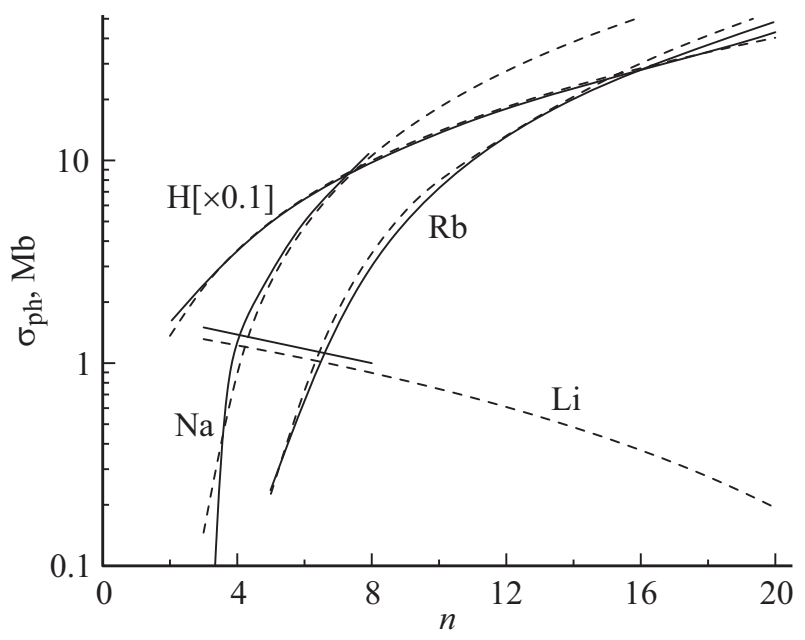

Рис. 2. Пороговые значения сечений фотоионизации $\sigma_{p h}$ для $s$-серий в зависимости от главного квантового числа $n$ ридберговских состояний атомов $\mathrm{H}, \mathrm{Na}, \mathrm{Rb}$ и Li. Квантовомеханические данные [26] отображены непрерывными кривыми, наши квазиклассические результаты представлены штриховыми линиями. 
состояний щелочных атомов приведены в табл. 1 [24,25]. На рисунке сечения $\sigma_{p h}$ представлены как функции главного квантового числа $n$ для $s$-серий атомов водорода, лития, натрия и рубидия. Квазиклассические результаты повторяют квантово-механические вычисления $\sigma_{p h}[26] \mathrm{c}$ ошибкой не больше $10 \%$ (для больших $n^{*}$ погрешность оказывается меньше 7\%), которая соответствует 5\%-точности расчета радиальных интегралов для связанносвободных переходов. Результирующую погрешность приведенных ниже результатов для автоионизационных ширин $\tilde{\Gamma}_{N}(2)$ можно оценить как $10 \%+2 \cdot 2 \%=14 \%$.

\section{3. Результаты расчетов автоионизационных ширин ПИ и их обсуждение}

На рис. 3 представлены полученные нами данные для приведенных автоионизационных ширин $\tilde{\Gamma}_{N}$ (2) ридберговской квазимолекулы. Поверхности рисунка иллюстрируют поведение $\tilde{\Gamma}_{N}$ при варьировании главных квантовых чисел $n_{i}, n_{d}$ для пары атомов водорода $(a)$ или лития $(b)$ при неизменных орбитальных квантовых числах $l_{d}, l_{i}$ (орбитальной конфигурации) пары. Отметим, что рассмотренный в работе [12] случай
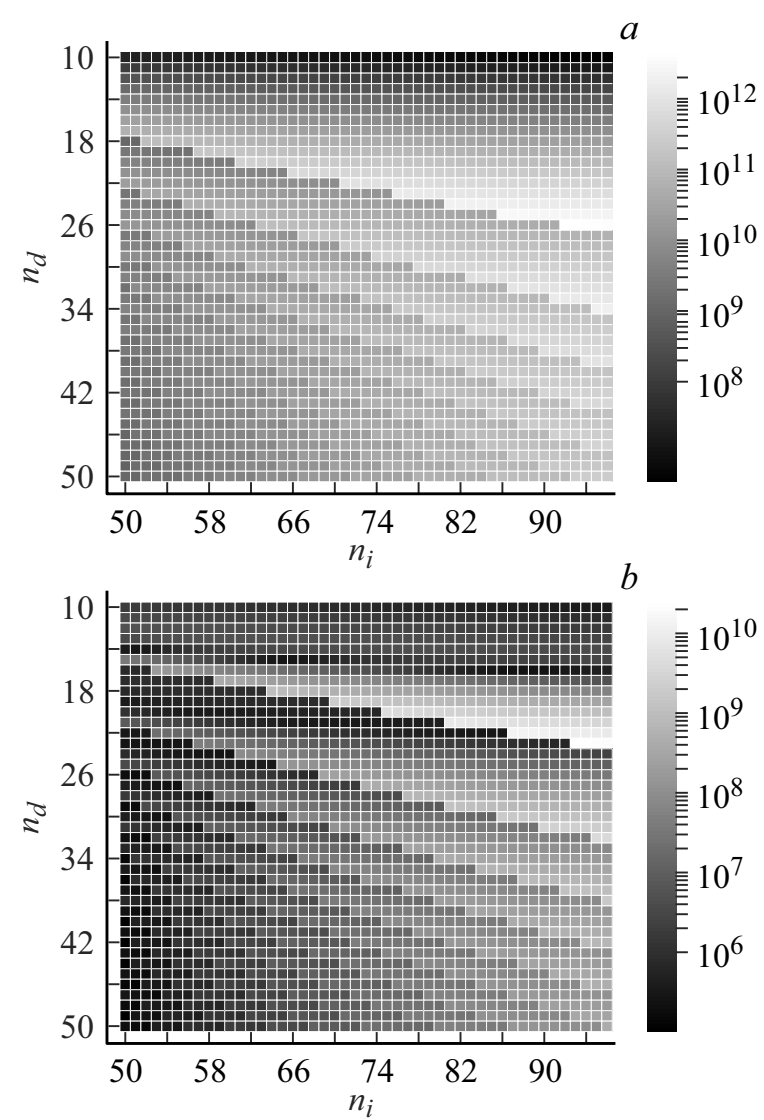

Рис. 3. Зависимость приведенной автоионизационной ширины $\tilde{\Gamma}_{N}=R^{6} \Gamma_{N}$ (at.un.) от главных квантовых чисел $n_{d}, n_{i}$ двухатомной квазимолекулы. Рассмотрены случаи $s-s\left(l_{d}=0, l_{i}=0\right)$ пар атомов водорода $(a)$ и лития $(b)$.

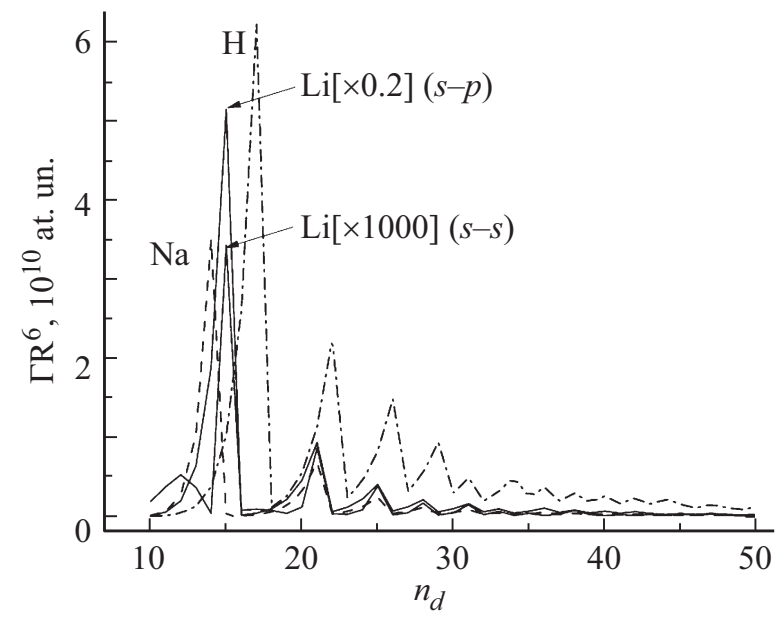

Рис. 4. Приведенные автоионизационные ширины $\tilde{\Gamma}_{N}$ (2) для случая $s-s$-конфигураций $\left(l_{d}=0, l_{i}=0\right)$ пар атомов $\mathrm{H}$ (штрихпунктир), Li (сплошная) и $\mathrm{Na}$ (штрих) с фиксированным значением $n_{i}=50$. Для сравнения приведена также $s-p$ конфигурация $\left(l_{d}=0, l_{i}=1\right)$ в случае $\mathrm{Li}$.

Таблица 1. Квантовые дефекты $\delta_{l}$ для $s$-, p-серий атомов водорода и щелочных металлов [24,25]

\begin{tabular}{c|c|c|c|c|c|c}
\hline & $\mathrm{H}$ & $\mathrm{Li}$ & $\mathrm{Na}$ & $\mathrm{K}$ & $\mathrm{Rb}$ & $\mathrm{Cs}$ \\
\hline$s$ & 0.00 & 0.40 & 1.35 & 2.19 & 3.13 & 4.05 \\
$p$ & 0.00 & 0.047 & 0.85 & 1.71 & 2.65 & 3.57 \\
$\delta_{s}-\delta_{p}$ & 0.00 & 0.353 & 0.50 & 0.48 & 0.48 & 0.48
\end{tabular}

водородных атомов характеризуется незначительной зависимостью $\tilde{\Gamma}_{N}$ от $\left(l_{d}, l_{i}\right)$-конфигурации. Литию, как и другим атомам щелочных металлов, напротив, присуще большее многообразие возможностей. Например, рис. 4 демонстрирует падение ( на три порядка) пикового значения $\max \tilde{\Gamma}_{N}(s, s)$ для $s-s$-пар и рост (до $\sim$ четырех раз) значения $\max \tilde{\Gamma}_{N}(s, p)$ для $s-p$-пар Li относительно $\max \tilde{\Gamma}_{N}(s, s)$ водородных пар. Падение $\tilde{\Gamma}_{N}(s, s)$ обусловлено аномально малыми значениями сечений $\sigma_{p h}^{(s)}$ фотоионизации $s$-состояний Li [26] (рис. 2), в то время как рост экстремальных пиков $\tilde{\Gamma}_{N}(s, p)$ обязан увеличению дипольных моментов для связанных $n_{d} s \rightarrow n_{d}^{\prime} p$ переходов при стандартных („водородных“) величинах $\sigma_{p h}^{(p)}$ для $p$-состояний Li [26].

На рис. 3,4 обращает на себя внимание четко прописанная осцилляционная структура $\tilde{\Gamma}_{N}$ при вариациях как $n_{d}$, так и $n_{i}$, что выражается в горноподобном виде поверхностей $\tilde{\Gamma}_{N}$ : цепь хребтов (пики) разделены глубокими долинами (минимумы). Эти осцилляции относятся к характерным особенностям ПИ и обусловлены нелинейной зависимостью входящих в соотношение (2) параметров оптических переходов от квантовых чисел атомной пары. Хорошо известным свойством как дипольных матричных элементов $D_{N_{D}^{\prime} N_{D}}\left(\Gamma_{d}\right)$, так и сечений фотоионизации $\sigma_{p h}\left(\varepsilon_{i}^{\prime}\right)$ (где $\gamma_{d}=\left|n_{d}^{\prime *}-n_{d}^{*}\right|-\mathrm{cм}$. обозначения на рис. 1) является быстрое падение их значений 
при увеличении аргументов $[12,19,26]$. Поэтому возникновение пиков $\tilde{\Gamma}_{N}$ соответствует реализации пороговых значений $\varepsilon_{i}^{\prime}=0$, что на рис. 1 означает наложение реального состояния $\tilde{n}_{d}^{\prime} l_{d}^{\prime}$ на виртуальный уровень $n_{\mathrm{th}} l_{d}^{\prime}$. Расхождение между $\tilde{n}_{d}^{\prime}$ и $n_{\text {th }}$ приводит к падению, а их сближение к росту $\tilde{\Gamma}_{N}$, т.е. к осцилляциям.

\section{1. Оптимальная асимметричная пара}

Характер рельефа поверхностей рис. 3 указывает на возникновение парадоксального явления, противоречащего классическим интуитивным представлениям о квантовых состояниях. Чем больше размеры атомов $\left(\sim n^{2}\right)$, тем большими должны быть ассоциированные с ними дипольные моменты. Тем эффективнее должны быть стимулированные диполь-дипольным взаимодействием процессы, в частности, ионизация. Для почти симметричных $\left(n_{d} \approx n_{i}\right)$ соударений эти ожидания выполняются: одновременный рост главных квантовых чисел атомных пар сопровождается, согласно формуле (1), степенным ростом ширины $\tilde{\Gamma}_{N}$.

При фиксированных размерах $i$-атома ситуация, однако, меняется кардинальным образом. На рис. 4 прослеживается значительное увеличение $\tilde{\Gamma}_{N}$ при схлопывании размеров девозбуждающегося $d$-атома для различных орбитальных конфигураций пар атомов H, Na и Li. Это чисто квантовый эффект [12], обусловленный уменьшением „в среднем“ аргументов $\gamma_{d}$ и $\varepsilon_{i}^{\prime}$ радиационных констант, входящих в соотношение (2), по мере уменьшения $n_{d}$. Для каждого вида атома щелочного металла существует оптимальное значение $n_{d}^{\text {(opt) }}\left(n_{i}\right)$, при котором возникает максимальный пик $\Gamma_{n_{d} n_{i}}^{(\text {ot) }}$ приведенной ширины пары. Факт появления оптимальной пары обусловлен наложением двух условий: (i) реализацией порогового значения $\varepsilon_{i}^{\prime}=0$ для сечения фотоионизации, (ii) реализацией минимально возможного значения $\gamma_{d}=\left|n_{d}^{\prime *}-n_{d}^{*}\right|$ (которое тем не менее должно быть строго больше нуля). Математическая формулировка этих условий сводится к требованию (рис. 1) равенств энергии связи $\varepsilon_{0}=1 /\left(2 n_{i}^{* 2}\right)$ ионизующегося $i$-атома и энергии перехода $\Delta \varepsilon \approx \gamma / n_{d}^{* 3} d$-атома из начального состояния $n_{d} l_{d}$ в ближайшее к нему состояние $l_{d}^{\prime}$-серии:

$$
\begin{gathered}
\frac{\gamma_{d}^{(\mathrm{min})}}{\left(n_{d}^{\text {(opt) }}-\delta_{l_{d}}\right)^{3}}=\frac{1}{2\left(n_{i}-\delta_{l_{i}}\right)^{2}}, \\
0<\gamma_{d}^{(\mathrm{min})}=\left\{\begin{array}{lc}
\min (m-|\Delta \delta|) & \text { при } l_{d}^{\prime}-l_{d}=1 \\
\min (m+|\Delta \delta|) & \text { при } l_{d}^{\prime}-l_{d}=-1
\end{array}\right\} .
\end{gathered}
$$

Здесь $m=\Delta n$ пробегает целые значения, а $\Delta \delta=\delta_{l_{d}^{\prime}}-\delta_{l_{d}}$. Если решение $z=n_{d}^{\text {(opt) }}$ уравнения (6) является рациональным числом, то в качестве $n_{d}^{\text {(opt) }}$ следует брать ближайшее к $z$ целое число, не превосходящее $z$.

В табл. 2 сравниваются значения $n_{d}^{\text {(opt) }}$, полученные с помощью аналитического соотношения (6) и численным счетом, для $s-s$-, $s-p$-пар атомов щелочных металлов.

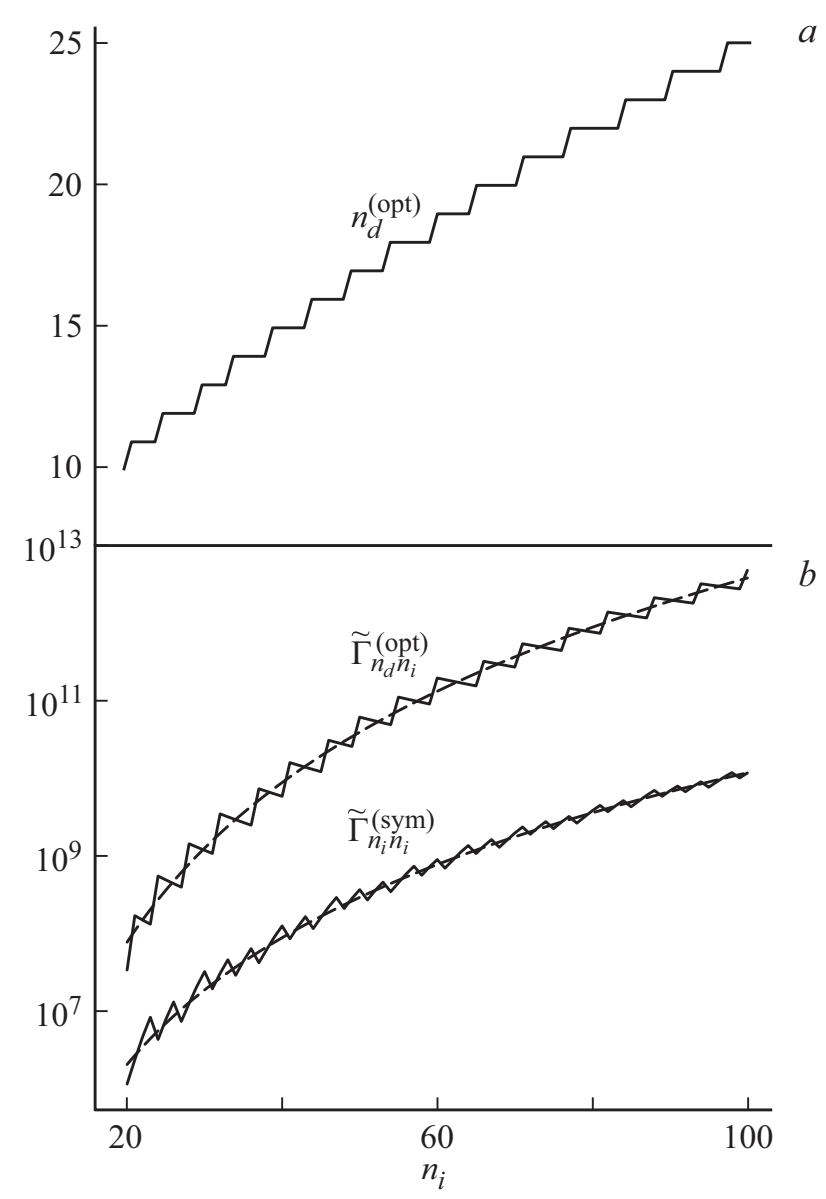

Рис. 5. (а) Главное квантовое число $n_{d}^{(\mathrm{opt})}(6)$ девозбуждающегося атома для оптимальной пары $\left(n_{i}, n_{d}^{\text {(opt) }}\right)$ и соответствующая ей приведенная ширина ПИ $\tilde{\Gamma}_{n_{d} n_{i}}^{(\mathrm{opt})}(b)$ как функции квантового числа $n_{i}$ ионизирующегося атома. Показана также ширина $\tilde{\Gamma}_{n_{i} n_{i}}^{(\mathrm{sim})}$ для симметричной пары $\left(n_{i}, n_{d}=n_{i}\right)$. Рассмотрен случай $s-s\left(l_{d}=0, l_{i}=0\right)$-конфигурации атомов цезия. Штриховые кривые получены с помощью аналитических соотношений (7), (8).

Одновременно представлены значения максимальных пиков $\Gamma_{n_{d} n_{i}}^{(\mathrm{opt})}$ автоионизационных ширин для оптимальных пар $n_{i}, n_{d}^{\text {(opt) }}$ с $n_{i}=50$. Последняя строка табл. 2 указывает на выигрыш $\Lambda\left(n_{i}\right)=\Gamma_{n_{d} n_{i}}^{(\mathrm{opt})} / \Gamma_{n_{d} n_{i}}^{(\mathrm{sym})}$ в скорости ПИ, когда первоначальная симметричная пара $\left(n_{d}=n_{i} n_{i}\right)$ заменяется на оптимальную пару $\left(n_{d}^{\text {(opt })}, n_{i}\right)$. Для атома водорода этот выигрыш приблизительно равен $n_{i}$ [12] (см. соотношение (1)), что хорошо согласуется с расчетной величиной $\Lambda\left(n_{i}=50\right)$ таблицы. Отметим, что для всех атомов щелочных металлов значения $\Lambda\left(n_{i}=50\right)$ существенно превышают эталонную „водородную“ величину 50 , а сам выигрыш $\Lambda$ имеет тенденцию к увеличению вместе с ростом разности квантовых дефектов серий.

Рис. 5 демонстрирует поведение характеристик оптимальной пары $\left(n_{d}^{(\text {opt })}, n_{i}\right)$ при увеличении главного квантового числа $n_{i}$. Хорошо виден существенный рост эффективности ПИ для пары $\left(n_{d}^{(\mathrm{opt})}, n_{i}\right)$ по сравнению 
Таблица 2. Оптимальные значения главных квантовых чисел $n_{d}^{(\mathrm{opt})}$ и приведенных автоионизационных ширин $\tilde{\Gamma}_{n_{d} n_{i}}^{\text {(opt }}$ для $s-s$ $\left(l_{d}=0, l_{i}=0\right)$ - и $s-p$ - $\left(l_{d}=0, l_{i}=1\right)$-конфигураций атомных пар с фиксированным значением $n_{i}=50$

\begin{tabular}{c|c|c|c|c|c|c|c}
\hline \multirow{2}{*}{$\gamma_{d}^{(\text {min) }}$} & $\mathrm{H}, s-s$ & $\mathrm{Li}, s-p$ & $\mathrm{Li}, s-s$ & $\mathrm{Na}, s-s$ & $\mathrm{~K}, s-s$ & $\mathrm{Rb}, s-s$ & $\mathrm{Cs}, s-s$ \\
\cline { 2 - 8 } & 1 & 0.647 & 0.647 & 0.50 & 0.52 & 0.52 & 0.52 \\
\hline$z(6)$ & 17.1 & 15.2 & 15.1 & 14.6 & 15.6 & 16.3 & 17.0 \\
$n_{d}^{(\text {opt) }}(6)$ & 17 & 15 & 15 & 14 & 15 & 16 & 17 \\
\hline$n_{d}^{\text {(opt) }}($ численный счет) & 17 & 15 & 15 & 14 & 15 & 16 & 17 \\
\hline$\tilde{\Gamma}_{n_{d} n_{i}}^{\text {(opt) }} / 10^{10}$ & 6.24 & 25.7 & $3.43 / 10^{3}$ & 3.50 & 2.29 & 3.35 & 3.94 \\
\hline$\tilde{\Gamma}_{n_{d} n_{i}}^{\text {(opt) }} / \tilde{\Gamma}_{n_{i} n_{i}}^{\text {(sym) }}$ & 52.4 & 184 & 158 & 257 & 266 & 482 & 364
\end{tabular}

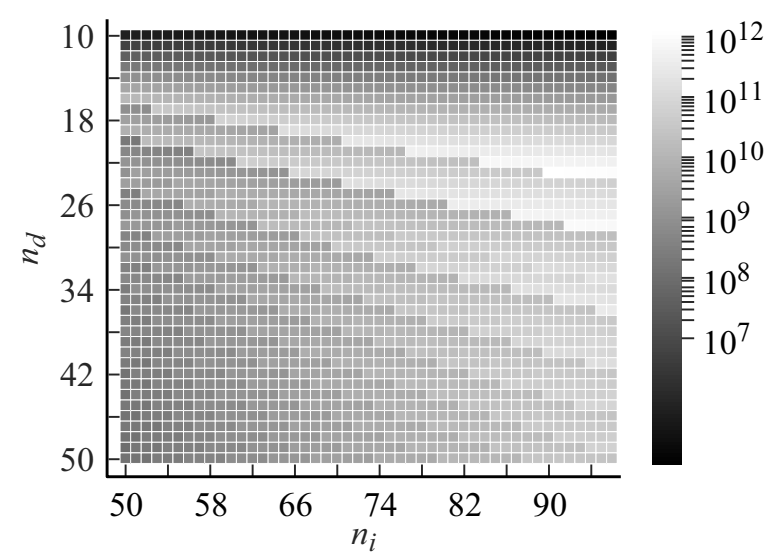

Рис. 6. Зависимость приведенной автоионизационной ширины $\tilde{\Gamma}_{N}=R^{6} \Gamma_{N}(R)$ (at.un.) от главных квантовых чисел $n_{d}, n_{i}$ для $p-s$-пары $\left(l_{d}=1, l_{i}=0\right)$ атомов цезия.

с симметричной парой $n_{i} n_{i}$. В окрестности $n_{i}=100$ выигрыш $\Lambda$ достигает $\sim 10^{3}$ при замене симметричной пары на оптимальную.

Приведенные результаты относятся к наиболее простому случаю пар, у которых $l_{d}=0$, т.е. оптические переходы вниз реализуются единственной атомной $p$ серией. При рассмотрении других типов пар, например $p-s \quad\left(l_{d}=1, l_{i}=0\right), d$-атом совершает двойные переходы $\quad$ в $s\left(l_{d} \rightarrow l_{d}^{\prime}=0\right)$ - или $d\left(l_{d} \rightarrow l_{d}^{\prime}=2\right)$-состояния атомных серий. При этом в силу различных квантовых дефектов для каждого из указанных переходов существует своя индивидуальная оптимальная пара (6), что может привести к появлению двух различных близко расположенных экстремальных пиков в области $n_{d} \ll n_{i}$. Реализация указанной возможности продемонстрирована на рис. 6 на примере $p-s$-пары $\left(l_{d}=1, l_{i}=0\right)$ атомов цезия.

\section{2. Аналитические оценки}

Ридберговские состояния атомов в условиях лабораторного эксперимента отвечают большим главным квантовым числам $n$ с типичными значениями 50-100.
Этот факт позволяет найти (Приложение) простые формулы (П10), (П11) для расчета радиальных матричных элементов и сечений фотоионизации. Беря их за основу, удается получить аналитические оценки эффективности процессов ПИ для частных, но практически важных случаев автоионизационных ширин $\Gamma_{N^{\prime}}$ симметричных/оптимальных пар. Опуская довольно громоздкие выкладки, которые будут представлены в будущей публикации [27], приведем конечные результаты:

$$
\begin{gathered}
\left\langle\tilde{\Gamma}_{n_{i} n_{i}}^{(\mathrm{sim})}\right\rangle\left(n_{i}\right)=\Pi_{l_{d} l_{i}}^{(\mathrm{sim})} \frac{1+34 \sqrt{2} /\left(3 n_{i}^{*}\right)}{\left(1+4 \sqrt{2} \Xi_{l_{d} l_{i}}^{(\mathrm{sim})} / n_{i}^{*}\right)^{17 / 3}} n_{i}^{* 16 / 3}, \\
\left\langle\tilde{\Gamma}_{n_{d} n_{i}}^{(\mathrm{opt})}\right\rangle\left(n_{i}\right)=\Pi_{l_{d} l_{i}}^{(\mathrm{opt})} \frac{\left(1-\Xi_{l_{d} l_{i}}^{(\mathrm{opt})} n_{i}^{* 2 / 3}\right)^{4}}{\left(1+3 \Xi_{l_{d} l_{i}}^{(\mathrm{opt})} / n_{i}^{* 2 / 3}\right)^{10 / 3}} n_{i}^{* 19 / 3} .
\end{gathered}
$$

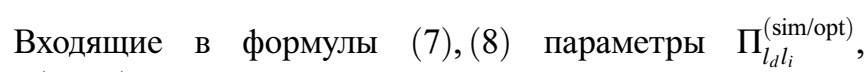
$\Xi_{l_{d} l_{i}}^{(\text {sim/opt })}$ являются функциями только орбитальных $\left\{l_{d}, l_{i}\right\}$ конфигураций пар. Первый тип параметров (П) регулирует численные значения констант скоростей ПИ. В случае атома водорода параметры $\Pi_{l_{d} l_{i}}^{(\mathrm{sim} / \mathrm{opt})}$ равны

$$
\begin{gathered}
\Pi_{l_{d} l_{i}}^{(\mathrm{sim})}=\frac{3^{2 / 3} 2^{19 / 3}}{17 \pi^{3}} \Gamma^{4}(2 / 3)=1.070, \\
\Pi_{l_{d} l_{i}}^{\text {opt })}=\frac{2^{18 / 3} \Gamma^{2}(2 / 3)}{3^{2 / 3} \pi}\left(\tilde{\mathfrak{I}}_{1}(1)\right)^{2}=1.899,
\end{gathered}
$$

т.е. оказываются константами, не зависящими от орбитальных квантовых чисел. Функция $\tilde{\mathfrak{I}}_{\gamma}(x)$ является производной от функции Ангера и определена в Приложении соотношением (П4).

Второй тип параметров $(\Xi)$, являющийся безразмерным, отвечает за осцилляционную структуру (рис. 5) констант скоростей ПИ. Графики функций, соответствующих значениям $\Xi_{l_{d} l_{i}}^{\text {(sim/opt) }} \geq 1$, проходят через максимумы функций $\tilde{\Gamma}_{n_{d} n_{i}}^{(\mathrm{simpt})}\left(n_{i}\right)$ (рис. 7). Значения с $\Xi_{l_{d} l_{i}}^{(\mathrm{sipt})} \leq 0$ позволяют описывать положения минимумов, как это видно на рис. 7. Если интересоваться усредненными по осцилляциям значениям пеннинговских ширин, то в первом приближении можно положить $\Xi_{l_{d} l_{i}}^{(\mathrm{sim} / \mathrm{opt})}=0.5$, 
Таблица 3. Значения параметров П, $\Xi$ для основных конфигураций $l_{d}-l_{i}$ атомных пар калия. Переводной коэффициент для значений параметров П: 1 at.un. $=0.906 \cdot 10^{-33} \mathrm{~cm}^{6} \mathrm{~s}^{-1}$

\begin{tabular}{|c|c|c|c|c|c|c|c|c|c|c|}
\hline$l_{d}-l_{i}$ & $s-s$ & $s-p$ & $s-d$ & $s-f$ & $s-g$ & $p-s$ & $p-p$ & $p-d$ & $p-f$ & $p-g$ \\
\hline$\Pi^{(\operatorname{sim})}$ & 0.111 & 0.124 & 0.163 & 0.591 & 0.562 & 0.113 & 0.129 & 0.154 & 0.604 & 0.543 \\
\hline$\Xi^{(\operatorname{sim})}$ & 0.69 & 0.58 & 0.12 & 0.07 & 0.13 & 0.88 & 0.78 & 0.05 & 0.23 & 0.12 \\
\hline$\Pi^{(\mathrm{opt})}$ & 2.10 & 2.20 & 2.63 & 10.9 & 9.95 & 1.80 & 1.51 & 1.80 & 6.99 & 6.371 \\
\hline \multirow[t]{2}{*}{$\Xi^{(\mathrm{opt})}$} & 0.98 & 0.79 & 0.42 & 0.57 & 0.60 & 1.29 & 0.77 & 0.44 & 0.50 & 0.49 \\
\hline & $d-s$ & $d-p$ & $d-d$ & $d-f$ & $d-g$ & $f-s$ & $f-p$ & $f-d$ & $f-f$ & $f-g$ \\
\hline$\Pi^{(\mathrm{sim})}$ & 0.178 & 0.199 & 0.243 & 0.948 & 0.856 & 0.120 & 0.135 & 0.164 & 0.658 & 0.601 \\
\hline$\Xi^{(\mathrm{sim})}$ & 1.28 & 1.19 & 0.42 & 0.59 & 0.54 & 1.37 & 1.29 & 0.50 & 0.78 & 0.75 \\
\hline$\Pi^{(\mathrm{opt})}$ & 0.840 & 0.970 & 4.40 & 4.31 & 0.807 & 0.679 & 0.712 & 3.26 & 2.84 & \\
\hline \multirow[t]{2}{*}{$\Xi^{(\mathrm{opt})}$} & 0.97 & 0.95 & 0.50 & 0.62 & 0.75 & 1.4 & 0.89 & 0.32 & 0.68 & 0.60 \\
\hline & $g-s$ & $g-p$ & $g-d$ & $g-f$ & $g-g$ & & & & & \\
\hline$\Pi^{(\operatorname{sim})}$ & 0.208 & 0.240 & 0.285 & 1.15 & 1.07 & & & & & \\
\hline$\Xi^{(\mathrm{sim})}$ & 0.98 & 0.99 & 0.11 & 0.42 & 0.44 & & & & & \\
\hline$\Pi^{(\text {opt })}$ & 0.4 & 0.45 & 0.453 & 1.76 & 1.65 & & & & & \\
\hline$\Xi^{(\mathrm{opt})}$ & 0.64 & 0.61 & 0.03 & 0.11 & 0.14 & & & & & \\
\hline
\end{tabular}

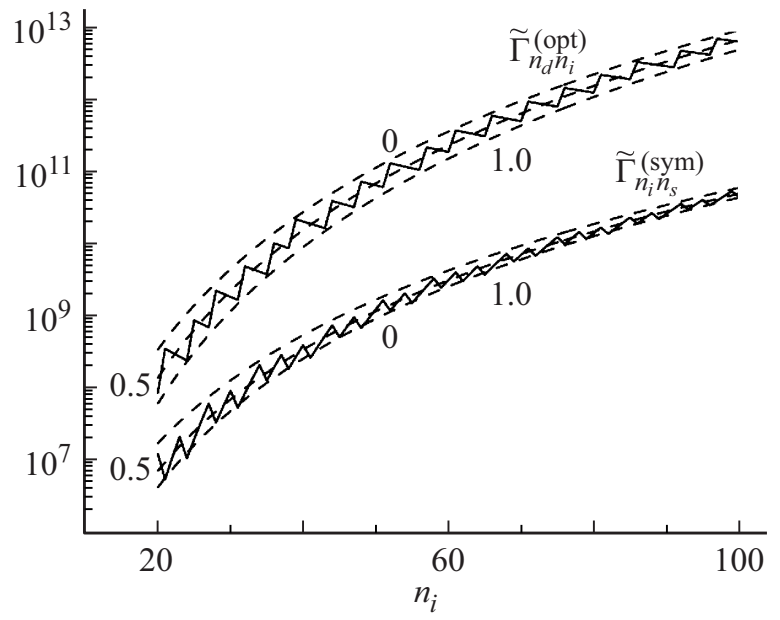

Рис. 7. Численные результаты расчетов (сплошные кривые) приведенных ширин АИ для оптимальных и симметричных $s-s \quad\left(l_{d}=0, l_{i}=0\right)$ пар атомов водорода. Аналитические результаты для $\tilde{\Gamma}_{n_{i} n_{i}}^{(\text {sim) }}$ (соотношение (7)) с параметрами $\Xi_{l_{d} l_{i}}^{(\text {sim) }}=0,0.5,1$ и для $\tilde{\Gamma}_{n_{d} n_{i}}^{\text {(opt) }}$ (соотношение $\left.(8)\right)$ с параметрами $\Xi_{l_{d} l_{i}}^{\text {(opt) }}=0,0.5,1$ показаны штриховыми кривыми, рядом с которыми проставлены численные значения параметров $\Xi$.

что хорошо согласуется с данными рис. 7 для атомов водорода.

В случае атомов щелочных металлов оба типа параметров П, $\Xi$, можно рассматривать как подгоночные, и использовать формулы (7), (8) для быстрых оценок автоионизационных ширин ПИ. О качестве подобных расчетов констант скоростей ПИ в „среднем“ можно судить по данным, представленным на рис. 5, где штриховые кривые представляют результаты аналитических расчетов пеннинговских ширин со следующими значениями параметров П и $\Xi$ для $s-s\left(l_{d}=0, l_{i}=0\right)$ пар атомов цезия: $\Pi_{s s}^{(\mathrm{sim})}=0.115, \Xi_{s s}^{(\mathrm{sim})}=0.6 ; \Pi_{s s}^{(\mathrm{opt})}=2.3$, $\Xi_{s s}^{(\mathrm{opt})}=0.7$.

В табл. 3 помещены в качестве примера значения параметров П, $\Xi$, для основных орбитальных конфигураций атомных пар калия. Виден большой разброс (до 25 раз) значений П, что указывает на существенную зависимость ПИ от орбитальных квантовых чисел атомарных пар. Стоит обратить внимание на конфигурации $s-f, s-g$, параметры П ${ }^{(\mathrm{opt})}$ для которых почти в пять раз превышают „эталонное“ водородное значение 1.9 (9). Как показано в [28], причиной подобной интенсификации процессов ПИ служит близость энергетических уровней $p$ - и $s$-серий к условиям двойного резонанса Штарка (резонанс Фёрстера (Förster)). Последний используется в качестве управляющего механизма для варьировании дальнодействующего межатомного взаимодействия и имеет многочисленные применения в прикладных задачах ридберговских сред [29].

\section{4. Заключение}

Процессы ПИ при участии двух холодных ридберговских щелочных атомов, возбужденных в разные квантовые состояния и испытывающих диполь-дипольное взаимодействие, обладают широким набором особенностей. В рамках квазиклассического приближения нами проведен анализ поведения автоионизационных ширин $\Gamma_{N_{1}, N_{2}}$ ПИ в зависимости от квантовых чисел $\left\{N_{d}, N_{i}\right\}$ $\left(N_{j}=n_{j} l_{j}\right)$ квазимолекулы, образованной атомами партнерами по взаимодействию. Для всех атомов щелочных металлов продемонстрировано существование оптимальных ридберговских пар $\left(n_{d}=n_{d}^{(\mathrm{opt})}, n_{i}\right)$, которые 
оказываются сильно асимметричными $\left(n_{d}^{\text {(opt })} \ll n_{i}\right)$ и вызывают взрывное увеличение скорости ПИ (на несколько порядков величин) по сравнению с симметричными парами $\left(n_{d}=n_{i} n_{i}\right)$. Это свойство делает ПИ важным источником образования первичных заряженных частиц при формировании холодной ридберговской плазмы. В отличие от „эталонного“ случая атомов водорода, рассмотренного в [15], различие в квантовых дефектах атомных серий для атомов щелочных металлов приводит к сильной и нерегулярной зависимости пеннинговских ширин $\Gamma_{N_{1}, N_{2}}$ от орбитальных квантовых чисел $\left(l_{d}, l_{i}\right)$ квазимолекулы. В частности, приведенные нами численные данные для атомных пар калия демонстрируют существенное влияние резонанса Фёрстера (Förster) на константы скоростей ПИ. Последние в окрестности резонанса испытывают пятикратное увеличение по сравнению с атомами водорода.

\section{Приложение. Квазиклассические представления радиальных интегралов}

Квазиклассические представления дипольных матричных элементов при оптических переходах $n l \rightarrow n^{\prime} l^{\prime}$ основаны на принципах соответствия Бора-Гейзенберга [20]. Для ридберговских $(n \gg 1)$ близколежащих квантовых состояний $\left(\left|n^{\prime *}-n^{*}\right|<10\right)$ радиальные матричные элементы $R_{n l}^{n^{\prime} l^{\prime}}$ определяются коэффициентами фурьеразложения классической электронной орбиты, которая ассоциируется со связанным квантовым состоянием $n l$. Соответствующие представления $R_{n l}^{n^{\prime} l^{\prime}}$ для щелочных атомов получены в работах [22,23,29]:

$$
\begin{gathered}
R_{n l}^{n^{\prime} l^{\prime}}=\frac{l_{\max }^{2}}{\Delta \varepsilon \sqrt{n^{* 3} n^{\prime * 3}}} G_{\Delta l}(\gamma), \quad \gamma=n^{\prime *}-n^{*}, \\
G_{\Delta l}(\gamma)=\frac{(-1)^{\Delta n} n_{a}^{* 2}}{l_{\max }^{2}}\left\{\frac { \operatorname { s i n } ( \pi \gamma ) } { \pi \gamma } \left[\gamma\left(1-e_{a}\right)\right.\right. \\
\left.-\Delta l \sqrt{1 / e_{a}^{2}-1}\right]-\tilde{\mathfrak{I}}_{\gamma}\left(-\gamma e_{a}\right) \\
\left.+\Delta l \sqrt{1 / e_{a}^{2}-1} \mathfrak{I}_{\gamma}\left(-\gamma e_{a}\right)\right\} .
\end{gathered}
$$

Символ $\Delta$ подразумевает разность физических величин, относящихся к конечному $\left(n^{\prime} l^{\prime}\right)$ и начальному $(n l)$ состояниям. Например, $\Delta n^{*}=n^{*}-n^{*} \equiv \gamma ; \Delta l=l^{\prime}-l$. Индекс , $a^{\text {“ } у к а з ы в а е т ~ н а ~ п р о м е ж у т о ч н ы и ̆ ~ п о ~ о т н о ш е н и ю ~}$ к состояниям $n l ; n^{\prime} l^{\prime}$ уровень $n_{a} l_{a}$, квазиклассические параметры которого были получены в работах [22,23]:

$$
\begin{gathered}
l_{a}=l_{\max }=\max \left\{l, l^{\prime}\right\}, \\
n_{a}^{* 3}=\frac{2 n^{* 2} n^{* 2}}{n^{*}+n^{\prime *}}, \quad e_{a}=\sqrt{1-l_{a}^{2} / n_{a}^{* 2}} .
\end{gathered}
$$

Входящие в (П2) функции являются функцией Ангера и ее производной:

$$
\mathfrak{I}_{\gamma}(x)=\frac{1}{\pi} \int_{0}^{\pi} d \xi \cos (\gamma \xi-x \sin \xi), \quad \tilde{\mathfrak{I}}_{\gamma}(x)=\frac{d}{d x} \mathfrak{I}_{\gamma}(x) .
$$

Как показывает сравнение результатов расчетов $R_{n l}^{n^{\prime} l^{\prime}}$ по формуле (П1) с квантово-механическими данными, погрешность (П1) составляет доли процента для $n_{a} \geq 10$ и единицы процента для $n_{a} \approx 3$ [22,29].

Формулы (П1), (П2) неудобны при работе с ,длинными“ $|\gamma| \gg 1$ переходами. В то же время наличие большого параметра $\gamma$ позволяет получить [23] асимптотическое представление для функции $G_{\Delta l}(\gamma)(П 2)$, что сводит (П1) к виду

$$
R_{n l}^{n^{\prime} l^{\prime}}=\frac{l_{\max }^{2}}{\Delta \varepsilon \sqrt{n^{* 3} n^{* 3}}} G_{\Delta l}^{(\mathrm{as})}(x), \quad x=\left(\Delta \varepsilon l_{\max }^{3} / 2\right)^{2 / 3} .
$$

Асимптотика $G_{\Delta l}^{\text {(as) }}$ является функцией нового аргумента $x$ (приведенной „частоты“ рассматриваемого перехода):

$$
\begin{aligned}
& G_{\Delta l}^{\mathrm{as})}(x)=-\frac{\sin (\pi \Delta \delta)}{\pi}\left(\frac{1}{2}+\frac{\Delta l}{l_{\max }}\left(\Lambda_{q}+1\right)\right) \\
& +\frac{1}{x \sqrt{\pi}} \tilde{\Phi}_{\Delta \delta}(x)+\left(\Delta l+\frac{\Lambda_{q}}{5 l_{\max }}\right) \sqrt{\frac{1}{\pi x}} \Phi_{\Delta \delta}(x),
\end{aligned}
$$

и выражается через модифицированную функцию Эйри $\Phi_{\Delta \delta}(x)$ и ее производную $\tilde{\Phi}_{\Delta \delta}(x)$ :

$$
\begin{gathered}
\Phi_{\Delta \delta}(x)=\frac{1}{\sqrt{\pi}} \int_{0}^{\infty} \cos \left(x \xi+\frac{\xi^{3}}{3}+\pi \Delta \delta\right) d \xi, \\
\tilde{\Phi}_{\Delta \delta}(x)=-\frac{d}{d x} \Phi_{\Delta \delta}(x) .
\end{gathered}
$$

Соотношение (П6) содержит разность квантовых дефектов $\Delta \delta=\delta_{l^{\prime}}-\delta_{l}$, а также квантовый фактор $\Lambda_{q}=\left(\varepsilon^{\prime}+\varepsilon\right) / \Delta \varepsilon$, который учитывает квантовые поправки более высоких порядков в квазиклассическом приближении [30,31].

Отметим, что вывод асимптотики (П5) предполагает положительность разности $\gamma=n^{*}-n^{*}$, т. е. рассматриваются переходы, у которых конечный уровень всегда лежит выше исходного. В силу самосопряженности матричных элементов ситуация сводится к случаю $\gamma>0$ за счет перестановки уровней: $R_{n l}^{n^{\prime} l^{\prime}}=R_{n^{\prime} l^{\prime}}^{n l}$.

Важная особенность асимптотических формул (П5) заключается в возможности с неплохой $(\sim 5 \%)$ погрешностью получать значения радиальных интегралов (П1) даже в области параметра $\gamma \sim 1$. При численных расчетах пеннинговских ширин нами будет использоваться в соотношении (4) представление (П1) для $R_{n l}^{n^{\prime} l^{\prime}}$, если в индивидуальном переходе $N \rightarrow N^{\prime}$ параметр $|\gamma| \leq 4$, и представление (П5) в противном случае (т.е. при $|\gamma|>4)$.

В типичных условиях лабораторных экспериментов с холодными ридберговскими атомами главные квантовые числа $n \cong 50$, тогда как $l$ не превышает нескольких единиц. Это означает малость параметра $x \sim l_{\max } / n^{4 / 3}$ в (П5), что приводит к значительному упрощению функций Эйри:

$$
\lim _{x \rightarrow 0} \Phi_{\Delta \delta}(x)=\frac{\Gamma(1 / 3)}{\sqrt{\pi} 3^{2 / 3}} \sin \left(\frac{\pi}{3}-\pi \Delta \delta\right),
$$




$$
\lim _{x \rightarrow 0} \tilde{\Phi}_{\Delta \delta}(x)=\frac{\Gamma(2 / 3)}{\sqrt{\pi} 3^{1 / 3}} \sin \left(\frac{\pi}{3}+\pi \Delta \delta\right) .
$$

Здесь $Г(x)$ является Г-функцией Эйлера. Для малых $x$ доминирующим членом в функции $G_{\Delta l}^{(\text {as) }}(x)(П 6)$ становится второе слагаемое, и тогда соотношения (П5), (5) для радиального матричного элемента и сечения фотоионизации приобретают вид

$$
\begin{gathered}
R_{n_{d} l_{d}}^{n_{d}^{\prime} l_{d}^{\prime}} \cong \frac{2^{2 / 3} \Gamma(2 / 3)}{3^{1 / 3} \pi} \frac{\sin (\pi / 3-\pi \Delta \delta)}{\omega_{d}^{5 / 3} \sqrt{n_{d}^{* 3} n_{d}^{\prime * 3}}} \\
\sigma_{p h}\left(n_{i} \rightarrow \varepsilon_{i}^{\prime}\right) \cong \frac{2^{10 / 3} \Gamma^{2}(2 / 3)}{3^{5 / 3} c} \frac{l_{\max }}{2 l_{i}+1} \frac{\sin ^{2}(\pi / 3+\pi \Delta \delta)}{\omega_{d}^{7 / 3} n_{i}^{* 3}},
\end{gathered}
$$

удобный для аналитических оценок констант скоростей широкого спектра физических процессов.

\section{Финансирование работы}

Работа выполнена при финансовой поддержке грантом Российского научного фонда № 18-12-00313.

\section{Конфликт интересов}

Авторы заявляют, что у них нет конфликта интересов.

\section{Список литературы}

[1] Shore Bruce W. Manipulating Quantum Structures Using Laser Pulses. Cambridge: University Press, 2011. 586 p. doi org/10.1017/cbo9780511675713

[2] Jones M.P.A., Marcassa L.G., Shaffer J.P. // J. Phys. B. 2017. V. 50. P. 060202. doi org/10.1088/1361-6455/aa5d06

[3] Saffman M., Walker T.G., Mфlmer K. // Rev. Mod. Phys. 2010. V. 82. P. 2313. doi org/10.1103/RevModPhys.82.2313

[4] Hofmann C.S., Günter G., Schempp H., Müller N.L., Faber A., Busche H., Robert-de-Saint-Vincent M., Weidemüller M. // Front. Phys. 2014. V. 9. P. 571. doi org/10.1007/s11467-0130396-7

[5] Pillet P., Gallagher T.F. // J. Phys. B. 2016. V. 49. P. 174003. doi org/10.1088/0953-4075/49/17/174003

[6] Reetz-Lamour M., Amthor T., Deiglmayr J., Westermann S., Singer K., de Oliveira A.L., Marcassa L.G., Weidemüller M. // Fortschr. Phys. 2006. V. 54. P. 776. doi org/10.1002/prop.200610318

[7] Рябцев И.И., Бетеров И.И., Третьяков Д.Б., Энтин В.М., Якшина Е.А. // УФН. 2016. Т. 186. С. 206; Ryabtsev I.I., Beterov I.I., Tretyakov D.B., Entin V.M., Yakshina E.A. // Physics-Uspekhi. 2016. V. 59. P. 196. doi org/10.3367/UFNe.0186.201602k.0206

[8] Lyon M., Rolston S.L. // Rep. Prog. Phys. 2017. V. 80. P. 017001. doi org/10.1088/0034-4885/80/1/017001

[9] Tanner P.J., Han J., Shuman E.S., Gallagher T.F. // Phys. Rev. Lett. 2008. V. 100. P. 043002. doi org/10.1103/PhysRevLett.100.043002

[10] Graham W.G., Fritsch W., Hahn Y., Tanis J.A. Recombination of Atomic Ions. Springer Science \& Business Media, 2012. $345 \mathrm{p}$.
[11] Beterov I.I., Tretyakov D.B., Ryabtsev I.I., Entin V.M., Ekers A., Bezuglov N.N. // New J. Phys. 2009. V. 11. P. 013052. doi org/10.1088/1367-2630/11/1/013052

[12] Efimov D.K., Miculis K., Bezuglov N.N., Ekers A. // J. Phys. B. 2016. V. 49. P. 125302. doi org/10.1088/09534075/49/12/125302

[13] Klyucharev A.N., Vujnović V. // Phys. Rep. 1990. V. 185. P. 55. doi org/10.1016/0370-1573(90)90112-F

[14] Ryabtsev I.I., Tretyakov D.B., Beterov I.I., Bezuglov N.N., Miculis K., Ekers A. // J. Phys. B. 2005. V. 38. P. S17. doi org/10.1088/0953-4075/38/2/002

[15] Klyucharev A.N., Bezuglov N.N., Matveev A.A., Mihajlov A.A., Ignjatović L.M., Dimitrijević M.S. // New Astron. Rev. 2007. V. 51. V. 547. doi org/10.1016/j.newar.2007.05.001

[16] Amthor T., Denskat J., Giese C., Bezuglov N.N., Ekers A., Cederbaum L., Weidemüller M. // Eur. Phys. J. D. 2009. V. 53. P. 329. doi org/10.1140/epjd/e2009-00119-4

[17] Katsuura K.J. // Chem. Phys. 1965. V. 43. P. 4149. doi org/10.1063/1.1696654

[18] Смирнов Б.М. // УФН. 1981. Т. 133. Р. 570; Smirnov B.M. // Soviet Physics Uspekhi. 1981. V. 24. P. 251.

[19] Собельман И.И. Введение в теорию атомных спектров. М.: Наука, 1977. 320 с.; Sobelman I.I. Atomic Spectra and Radiative Transitions. Berlin, Heidelberg: Springer, 1992. 356 p. doi org/10.1007/978-3-642-76907-8

[20] Ландау Л.Д., Лифиии Е.М. Квантовая механика. М.: Наука, 1989. 768 c.; Landau L.D., Lifshitz E.M. Quantum Mechanics. Oxford: Pergamon, 1977. 688 p.

[21] Delone N.B., Goreslavsky S.P., Krainov V.P. // J. Phys. B. 1994. V. 27. P. 4403. doi org/10.1088/0953-4075/27/19/004

[22] D'yachkov L.G., Pankratov P.M. // J. Phys. B. 1994. V. 27. P. 461. doi org/10.1088/0953-4075/27/3/012

[23] Безуглов Н.Н., Бородин В.М. // Опт. и спектр. 1999. Т. 86. № 4. C. 533; Bezuglov N.N., Borodin V.M. // Opt. Spectr. 1999. V. 86. P. 467.

[24] Snitchler G.L., Watson D.K. // J. Phys. B. 1986. V. 19. P. 259 268. http://stacks.iop.org/0022-3700/19/i=3/a=009.

[25] Hezel T.P., Burkhardt C.E., Ciocca M., He L-W., Leventhal J.J. // Am. J. Phys. 1992. V. 60. P. 329. doi org/10.1119/1.16876

[26] Aymar M., Robaux O., Wane S. // J. Phys. B. 1984. V. 17. P. 993. http://stacks.iop.org/0022-3700/17/i=6/a =013

[27] Abo Zalam Alaa, Miculis K., Bruvelis M., Beterov I.I., Bezuglov N.N., Klyucharev A.N., Ekers A. // J. Phys. B. In progress.

[28] Paris-Mandoki A., Gorniaczyk H., Tresp C., Mirgorodskiy I., Hofferberth S. // J. Phys. B. 2016. V. 49. P. 164001. doi org/10.1088/0953-4075/49/16/164001

[29] Давыдкин В.А., Зон Б.А. // Опт. и спектр. 1981. Т. 51. В. 1. C. 25; Davydkin V.N., Zon V.A. // Opt. Spectr. 1981. V. 51. P. 25.

[30] Burgess A. // Mon. Not. R. Astron. Soc. 1958. V. 118. P. 477. doi org $/ 10.1093 / \mathrm{mnras} / 118.5 .477$

[31] D'yachkov L.G., Pankratov P.M. // J. Phys. B. 1994. V. 27. P. 461. doi org/10.1088/0953-4075/27/3/012 